\title{
Modelo de Comunicação Molecular Multiportadora com Ruído Intracelular e Intercelular
}

\author{
Ligia F. Borges ${ }^{1}$, Michael T. Barros ${ }^{2}$, Michele Nogueira ${ }^{1}$ \\ ${ }^{1}$ Núcleo de Redes sem Fio e Redes Avançadas (NR2) \\ Universidade Federal do Paraná (UFPR) \\ ${ }^{2}$ Faculty of Medicine and Health Technology \\ Tampere University \\ \{lfborges, michele\}@inf.ufpr.br, michael.barros@tuni.fi
}

\begin{abstract}
Promising approaches for engineering data communication in nanonetwork are inspired by biological systems. However, molecular communications (MC) have poor performance due to the stochastic propagation of data and the excess noise in the environment. Noise causes errors in communication and compromises network performance. Thus, it is necessary to characterize accurately and model the sources of noises in the MC systems. This paper investigate intracellular and intercellular noises in a multi-carrier MC model for nanonetworks. The model was used to evaluate the communication performance considering the noise through metrics such as channel capacity and path loss. The analysis allowed us to identify the behavior of intracellular noise and how it affects communication performance and can contribute to the development of networks.
\end{abstract}

Resumo. Abordagens promissoras para a engenharia da comunicação de dados para nanorredes são inspiradas nos sistemas biológicos, porém as comunicações moleculares possuem baixo desempenho devido à propagação estocástica dos dados e ao excesso de ruído no ambiente. A presença de ruído gera comunicações propensas a erros e comprometem o desempenho da rede. Desta forma, torna-se necessário caracterizar com precisão e modelar as fontes de ruídos da comunicação molecular. Este artigo investiga o ruído intracelular e intercelular em um modelo de comunicação molecular com multiportadora para nanorredes. O modelo é aplicado para avaliar uma nanorrede na presença de ruídos através de métricas clássicas como a capacidade do canal e perda (path loss). A análise permitiu identificar o comportamento do ruído intracelular e como o ruído intercelular afeta o desempenho da comunicação, contribuindo com direções para a construção das nanorredes.

\section{Introdução}

Várias das aplicações de nanotecnologia estão no campo da biomedicina. As nanomáquinas podem ser implantadas no corpo humano para monitorar a glicose e colesterol, para detectar a presença de agentes infectantes, ou para identificar tipos de câncer [Barros et al. 2018b, Akyildiz et al. 2015]. A comunicação entre esses dispositivos forma as nanorredes, contribuído com aplicações diversas como a entrega de medicamentos inteligentes, realizando a sua liberação dentro do corpo de forma mais precisa e 
gradual [Barros et al. 2018b]. Abordagens promissoras para a engenharia da comunicação de dados nas nanorredes são inspiradas nos sistemas biológicos [Barros et al. 2018a]. Um exemplo é a troca de informação entre células que baseia-se na propagação de moléculas por meio de processos bioquímicos e físicos seguindo o paradigma da comunicação molecular (CM). A sinalização celular tem sido uma referência para a codificação de informação, seguindo técnicas como On-Off Keying (OOK), com base na concentração de moléculas [Nakano et al. 2007]. Contudo, a comunicação molecular tem demonstrado baixo desempenho devido à propagação dos dados ocorrer de forma estocástica, bem como o e excesso de ruído no ambiente [Barros and Dey 2017].

A literatura das nanorredes baseadas em comunicação celular seguem uma abordagem de portadora única de informação, geralmente baseada na molécula de cálcio $\left(\mathrm{Ca}^{2+}\right)$. Isto traz avanços neste novo campo de pesquisa, mas a comunicação nessas redes ainda requer avanços significativos no seu desempenho. Assim, como ocorreu com na evolução das redes sem fio convencionais, uma alternativa é seguir uma abordagem de multiportadora para melhorar o desempenho na comunicação das nanorredes. Neste trabalho, advogamos por esta hipótese. Porém, caracterizar os ruídos intracelular e intercelular decorrentes da utilização de moléculas como multiportadoras dos dados é uma etapa de extrema relevância, mas ainda em aberto. Em um sistema de comunicação baseado em reação bioquímica, os ruídos externo e interno são inevitáveis [Yu et al. 2009]. A presença de ruído gera comunicações propensas a erros e comprometem desempenho da rede, tornando necessárias a sua caracterização com precisão e a modelagem das fontes de ruído.

Este artigo apresenta um modelo de comunicação molecular multiportadora com ruído intracelular e intercelular. O ruído interno (intracelular) é modelado de acordo com as constantes flutuações das concentrações de moléculas de $\mathrm{IP}_{3}$ e $\mathrm{Ca}^{2+}$ que ocorrem dentro das células devido aos eventos estocásticos das reações químicas. O ruído externo é decorrente da permeabilidade dos canais intercelulares, ele é gerado devido às flutuações na concentração de moléculas vindas das células vizinhas. A investigação do comportamento da comunicação foi aplicada no contexto de um tecido astrócito 3D, uma das células mais abundantes do sistema nervoso central. O modelo considera a diversidade de moléculas, onde cada tipo de molécula desempenha o papel de um canal. As reações celulares são desencadeadas por um algoritmo estocástico acoplado com processo de difusão intercelular e integra o modelo das junções comunicantes de Kilinc e Akan [Kilinc and Akan 2013] para simular o comportamento que permite a comunicação entre pares de células.

O modelo foi utilizado para a análise da concentração espaço-temporal das moléculas de inositol trifosfato $\left(\mathrm{IP}_{3}\right)$ e $\mathrm{Ca}^{2+}$, e como a sinalização intracelular (dentro da célula) e intercelular (entre células) determina a propagação do sinal nos tecidos dos astrócitos, considerando os princípios da transmissão multiportadora e o ruído intracelular e intercelular. Além da avaliação do modelo na presença de ruídos, foram analisadas a capacidade do canal e a perda (path loss). A análise permitiu identificar o comportamento do ruído intracelular e como o ruído intercelular afeta negativamente o desempenho da comunicação. Os resultados reafirmam que a cooperação entre os dois canais, ou seja, nesta caso as moléculas de $\mathrm{IP}_{3}$ e $\mathrm{Ca}^{2+}$, pode beneficiar a codificação e a transmissão de dados em comparação com o seu uso individual (portadora única) em nanorredes moleculares.

Este artigo procede da seguinte forma. A Seção 2 discute o trabalhos relacionados. A Seção 3 apresenta a modelagem da comunicação. A Seção 4 detalha a metodologia de 
avaliação e discute os resultados obtidos. A Seção 5 apresenta as conclusões.

\section{Trabalhos Relacionados}

Diferentes pesquisas focam na modelagem do canal de comunicação [Nakano and Liu 2010, Heren et al. 2013, Barros et al. 2015, Barros et al. 2018a, Borges et al. 2019]. Em [Nakano and Liu 2010], os autores aplicaram abordagens da teoria da informação para propor um modelo de capacidade de canal em que a molécula de $\mathrm{Ca}^{2+}$ é utilizada como portadora de informação. Os resultados mostram que a capacidade do canal apresenta valores inferiores a 0,3 bits mesmo para curtas faixas de transmissão. $\mathrm{O}$ modelo inclui a maior parte da dinâmica intercelular de $\mathrm{Ca}^{2+}$. Posteriormente, o modelo foi estendido em [Heren et al. 2013] e acrescentada a dinâmica intercelular de $\mathrm{Ca}^{2+}$ via estimulação pela molécula $\mathrm{IP}_{3}$. Os autores investigaram a capacidade do canal e mostraram a necessidade de estudos mais aprofundados sobre a variação da frequência de concentração e o desenvolvimento de modelos de ruído na comunicação molecular. Em [Barros et al. 2015], os autores modelaram o ruído em três tipos distintos de tecidos (astrócitos, epitelial e muscular). Entretanto, eles se concentraram em investigar o ruído gerado pela deformação do tecido na comunicação molecular baseada em $\mathrm{Ca}^{2+}$. Em [Borges et al. 2019], os autores modelaram o canal de comunicação de $\mathrm{IP}_{3}$ e compararam com os resultados de capacidade e ganho do canal obtido pelo canal de $\mathrm{Ca}^{2+}$.

Em [Yu et al. 2009] os autores investigaram o ruído interno causado pela oscilação de $\mathrm{Ca}^{2+}$ citosólico. Este trabalho foi estendido em [He et al. 2018], onde os autores analisaram o impacto do ruído da molécula de $\mathrm{Ca}^{2+}$ na funcionalidade dos comutadores de canal nas nanorredes de comunicação molecular. Em [Nakano and Liu 2010], os autores mostraram que as simulações baseadas na equação diferencial ordinária (ODE) com o algoritmo estocástico de Gillespie [Gillespie 1977] produzem uma variabilidade precisa das reações químicas e servem para estudar os efeitos do ruído causado pelo comportamento estocástico. As pesquisas da literatura para comunicação molecular focaram na investigação do ruído para nanorredes seguindo a abordagem de única portadora de informação por $\mathrm{Ca}^{2+}$, que traz avanços no campo de pesquisa, mas são ausentes estudos que caracterizem os ruídos utilizando diferentes tipos de moléculas como multiportadoras. Desta forma, este trabalho visa preencher uma lacuna através da caracterização dos ruídos internos e externos na comunicação molecular por multiportadoras.

\section{Modelos da Comunicação Molecular Multiportadora com Ruídos}

Esta seção apresenta os fundamentos das nanorredes (Subsec. 3.1) e descreve o sistema de comunicação molecular com suas entidades, fazendo um paralelo com um sistema de comunicação de dados convencional. Este sistema é o mesmo seguido por outros autores e por nós mesmos em outros trabalhos, como [Barros et al. 2015, Borges et al. 2019, Borges et al. 2020]. Na sequência, detalha-se o modelo proposto de comunicação multiportadora com ruídos para a sinalização de $\mathrm{IP}_{3} \mathrm{e} \mathrm{Ca}^{2+}$ em células astrócitos. São descritos os seguintes modelos: $(i)$ processo de difusão que captura a dinâmica espaço-temporal da sinalização intercelular; (ii) junções comunicantes que influenciam a direção de difusão intercelular; (iii) modelo 3D do tecido celular; $($ iv $)$ modelo do ruído intracelular; $(v)$ processo estocástico para o agendamento das reações intracelulares e intercelulares. 


\subsection{Visão geral das Nanorredes e da Comunicação Tratada}

Este trabalho foca na comunicação entre dois ou mais nanodispositivos através da comunicação molecular dentro dos tecidos. A comunicação molecular em células está fundada em caminhos de sinalização, i.e., cadeias de reações químicas que processam sinais de informações moduladas por características químicas, ex. concentração de moléculas, tipo e estado de energia, para propagá-los de uma fonte (ou nanomáquina transmissora) para um destino (ou nanomáquina receptora). Para explorar a diversidade molecular dentro dos tecidos celulares e fornecer análises matemáticas mais ricas, foram comparados os resultados de dois cenários diferentes: (i) independência entre os canais $\mathrm{IP}_{3} \mathrm{e} \mathrm{Ca}^{2+}$; (ii) não independência entre estes dois canais, seguindo a sinalização natural das moléculas.

A Figura 1 ilustra o sistema de comunicação molecular tratado, destacando os canais paralelos $\mathrm{Ca}^{2+}$ e $\mathrm{IP}_{3}$, adaptados dos princípios dos sistemas de transmissão multiportadora. A nanomáquina transmissora é uma célula sintética dependente das propriedades do canal molecular para definir como as informações são codificadas. Neste trabalho, a informação é codificada em termos da concentração de uma determinada molécula. As nanomáquinas transmissoras usam da modulação molecular OOK para transmitir moléculas em períodos de bit 1 (com uma determinada concentração) e não transmitir moléculas em períodos de bit 0 (concentração também é zero). Para canais independentes é considerada a sinalização celular natural de $\mathrm{IP}_{3} / \mathrm{Ca}^{2+}$. O canal compreende a propagação de $\mathrm{IP}_{3}$ e $\mathrm{Ca}^{2+}$. O modelo do canal considera os estágios de sinalização intracelular e intercelular. Dentro da célula (intracelular), existem várias reações químicas para regular a concentração de $\mathrm{Ca}^{2+}$. A comunicação entre células (intercelular) ocorre quando as junções comunicantes nas células se abrem e as moléculas de $\mathrm{IP}_{3}$ e $\mathrm{Ca}^{2+}$ se propagam através do citosol (i.e., o líquido que preenche o citoplasma celular) de uma célula para outra. Por usar tecidos do corpo humano como o canal de comunicação, este trabalho segue a propagação passiva, onde codifica-se a informação pela concentração da molécula na nanomáquina transmissora. A nanomáquina receptora é uma célula sintética que recebe as moléculas com informação e posteriormente a decodifica. Dentro da célula, existe um conjunto de receptores responsáveis pela adesão distribuída de moléculas e pela quantificação da concentração de moléculas recebidas. A decodificação é baseada no valor de concentração com um detector de limiar predefinido.

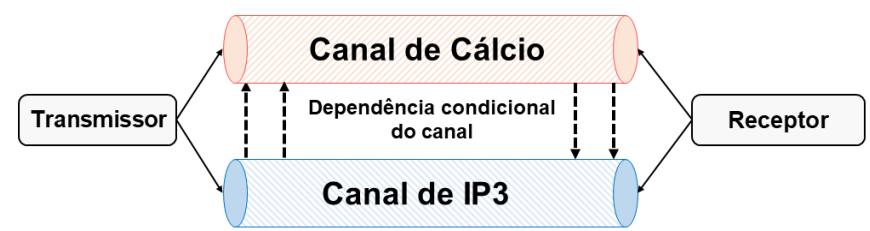

Figura 1. Sinalização molecular multiportadora de $\mathrm{IP}_{3}$ e $\mathrm{Ca}^{2+}$

\subsection{Modelo da Sinalização Celular de Cálcio e $\mathbf{I P}_{3}$}

Com base em observações experimentais, [Lavrentovich and Hemkin 2008] propuseram um modelo para descrever as oscilações de $\mathrm{Ca}^{2+}$ em células astrócitos. O modelo é composto pela representação de áreas de armazenamento ( $p o o l$ ) que incluem a concentração de $\mathrm{Ca}^{2+}$ no citosol $\left(C_{c y}\right)$ (Eq 1 ); a concentração de $\mathrm{Ca}^{2+}$ no retículo endoplasmático (estruturas membranosas compostas de sacos achatados e localizados no citosol da célula) 
$\left(C_{e r}\right)$ (Eq. 2); e a concentração de $\mathrm{IP}_{3}(I P)$ (Eq. 3). As equações a seguir definem matematicamente essas concentrações:

$$
\begin{gathered}
\frac{d C_{c y}}{d t}=X_{0}-k_{0} C_{c y}+X_{1}-X_{2}+l_{f}+W_{1} \\
\frac{d C_{e r}}{d t}=X_{2}-X_{1}-l_{f}, \\
\frac{d I P}{d t}=X_{3}-I P_{K_{\text {deg }}+W_{2}},
\end{gathered}
$$

onde, $X_{0}$ é o fluxo de cálcio do espaço extracelular para o citosol; $k_{0} C_{c y}$ é a taxa de efluxo de cálcio do citosol para o espaço extracelular; $l_{f}$ (i.e., $C_{c y}-C_{e r}$ ) é o fluxo de cálcio do retículo endoplasmático para o citosol; $I P_{K \text { deg }}$ é a degradação do $\mathrm{IP}_{3}$ por segundo; e $\mathrm{W}_{1}$ e $\mathrm{W}_{2}$ representam os ruídos intracelulares (citoplasma) para o $\mathrm{Ca}^{2+}$ e o $\mathrm{IP}_{3}$ conforme detalhado na Subseção 3.6. O termo $X_{1}$ (Eq. 4) modela o fluxo de cálcio do retículo endoplasmático para o citosol via estimulação de $\mathrm{IP}_{3}$. Este mecanismo afeta diretamente a concentração de cálcio no citosol. Ele é definido como:

$$
X_{1}=4 M_{3}\left(\frac{k_{A}^{n} C_{c y}^{n}}{\left(C_{c y}^{n}+k_{A}^{n}\right)\left(C_{c y}^{n}+k_{I}^{n}\right)}\right) \cdot\left(\frac{I P^{m}}{k_{I P}^{m}+I P^{m}}\right) \cdot l_{f} .
$$

O valor máximo de fluxo de cálcio no citosol é denotado por $M_{3} ; k_{A}$ e $k_{I}$ são as variáveis de ativação e inibição para do $\mathrm{IP}_{3} ; I P$ é a concentração de $\mathrm{IP}_{3}$ na célula; $m$ e $n$ são os coeficientes de Hill [Goldbeter et al. 1990]. Este coeficiente é amplamente utilizado na biologia molecular para descrever o grau de cooperatividade que um processo biológico tem sobre outro. Um coeficiente Hill de valor 1 indica ligação independente (ausência de cooperatividade) enquanto maior que 1 indica cooperatividade positiva. Os valores do coeficiente de Hill empregados neste estudo foram validados através de medições experimentais em [Lavrentovich and Hemkin 2008]. O efluxo de cálcio do retículo sarco-endoplasmático para o retículo endoplasmático é modelado como $X_{2}$, como apresenta a Eq. 5:

$$
X_{2}=M_{2} \frac{C_{e r}^{2}}{k_{2}^{2}+C_{e r}^{2}},
$$

onde o termo $M_{2}$ representa o fluxo máximo de cálcio nesse processo, $k$ é a constante de saturação da concentração citosólica de $\mathrm{Ca}^{2+}, C_{e r}$ é a concentração de $\mathrm{Ca}^{2+}$ no retículo endoplasmático e $X_{3}$ descreve a produção de $\mathrm{IP}_{3}$ pela proteína fosfolipases C (PLC):

$$
X_{3}=M_{p} \frac{C_{e r}^{2}}{C_{e r}^{2}+k_{p}^{2}} .
$$

onde $M_{p}$ é o fluxo máximo de $\mathrm{Ca}^{2+}$ nesse processo, $k$ é a constante de saturação da concentração citosólica de $\mathrm{Ca}^{2+}$, e $p$ é um coeficiente de Hill. Os valores dos parâmetros utilizados para a modelagem da comunicação são apresentados na Tabela 1.

\subsection{Modelagem da Junção Comunicante}

Uma junção comunicante é formada por duas partículas cilíndricas (conexônios), uma em cada par de célula. Cada conexônio é composto de seis proteínas conexinas. As junções comunicantes possuem propriedades biofísicas, como o controle de comporta e condutância, que permitem ou impedem o movimento de moléculas, como o $\mathrm{IP}_{3}$, e íons, como $\mathrm{Ca}^{2+}$, diretamente do citosol de uma célula para outra [Decrock et al. 2013]. Dessa 
forma, uma corrente gerada em qualquer célula poder fluir ao longo da junção comunicante e estimular outra célula a realizar uma determinada ação. Um modelo estocástico do comportamento dessas junções comunicantes foi introduzido em [Baigent et al. 1997] e é seguido neste trabalho. O modelo considera junções de abertura sensíveis à tensão que assumem dois estados de condutância para cada conexônio: aberto com alta condutância e fechado com baixa condutância. Com base nisto, consideram-se quatro combinações de estados de cada conexônio (Figura 2): (i) Estado $g_{1}-$ ambos canais estão em um estado de alta condutância (probabilidade $\mathrm{P}\left(g_{1}\right)$ ); (ii) Estado $g_{2}-\mathrm{o}$ primeiro conexônio está em um estado de alta condutância e o segundo (i.e., localizado na célula adjacente) em estado de baixa condutividade (a probabilidade é $\mathrm{P}\left(g_{2}\right)$ ); (iii) Estado $g_{3}$ - o primeiro conexônio está em um estado de baixa condutância e o outro está em um estado de alta condutância (probabilidade $\mathrm{P}\left(g_{3}\right)$ ); (iv) Estado $g_{4}$ - ambos canais estão em um estado de baixa condutância. Contudo, como a validação ex-

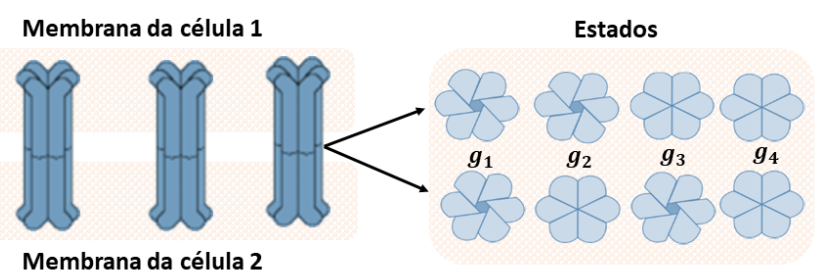

Figura 2. Junções comunicantes e possíveis estados

perimental desse modelo indicou baixa probabilidade de ocorrência do estado $g_{4}$ [Bukauskas et al. 2001], este estado não é considerado neste trabalho. Assim, as probabilidades dos estados para as junções comunicantes seguem $\mathrm{P}\left(g_{1}\right)+\mathrm{P}\left(g_{2}\right)+\mathrm{P}\left(g_{3}\right)=1$. Além disso, $g_{1}, g_{2}$ e $g_{3}$ estão inter-relacionadas da seguinte forma:

$$
\begin{gathered}
\frac{d g_{2}}{d t}=\beta_{1}(\vartheta j) \times g_{1}-\xi_{1}(\vartheta j) \times g_{3}, \\
\frac{d g_{3}}{d t}=\beta_{2}(\vartheta j) \times g_{1}-\xi_{2}(\vartheta j) \times g_{2},
\end{gathered}
$$

O controle da permeabilidade das junções comunicantes é mediado pela diferença de potência das membranas de duas células adjacentes $\left(\vartheta_{j}\right)$, a taxa de abertura da junção é classificada como $\xi$ e a taxa de fechamento como $\beta$. Os termos $\xi_{1}(\vartheta j), \xi_{2}\left(\vartheta_{j}\right), \beta_{1}\left(\vartheta_{j}\right)$, $\beta_{2}(\vartheta j)$ são definidos como: $\xi_{1}\left(\vartheta_{j}\right)=\lambda e^{-A \xi\left(\vartheta_{j}-\vartheta_{0}\right)} ; \xi_{2}\left(\vartheta_{j}\right)=\lambda e^{A \xi\left(\vartheta_{j}-\vartheta_{0}\right)} ; \beta_{1}\left(\vartheta_{j}\right)=$ $\lambda e^{A_{\beta}\left(\vartheta_{j}-\vartheta_{0}\right)} ; \beta_{2}\left(\vartheta_{j}\right)=\lambda e^{-A_{\beta}\left(\vartheta_{j}-\vartheta_{0}\right)}$. Sendo $\vartheta_{0}$ a tensão juncional na qual as taxas de abertura e fechamento das junções comunicantes têm o mesmo valor (i.e., $\xi=\beta$ ). $\lambda, A \xi$ e $A \beta$ são constantes e indicam a sensibilidade de uma junção comunicante à voltagem.

\subsection{Modelo da Difusão Molecular}

A difusão molecular segue um modelo que captura a dinâmica espaço-temporal da concentração de moléculas durante o processo de sinalização intercelular com base nos princípios da difusão mesoscópica [Nakano and Liu 2010].

$$
Z \Delta(i, j, k, n, m, l)=\frac{D_{\theta}}{v}\left(\left|Z_{n, m, l}-Z_{i, j, k}\right|\right) \cdot\left\{P\left(g_{1}\right), P\left(g_{2}\right), P\left(g_{3}\right)\right\},
$$

Em que $Z \Delta(i, j, k, n, m, l)$ é a diferença de concentração de moléculas entre um par de células vizinhas, sendo $i, j, k$ a posição da célula transmissora e $n, m, l$ a posição da célula receptora. Este valor é definido por $\frac{D_{\theta}}{v}\left(\left|Z_{n, m, l}-Z_{i, j, k}\right|\right)$ em que $D$ é o coeficiente de difusão para o $\mathrm{Ca}^{2+}$ ou $\mathrm{IP}_{3}$; $v$ é o volume da célula astrócito; $\left(\left|Z_{n, m, l}-Z_{i, j, k}\right|\right)$ é a diferença de concentração de moléculas entre a célula transmissora e a célula receptora. 


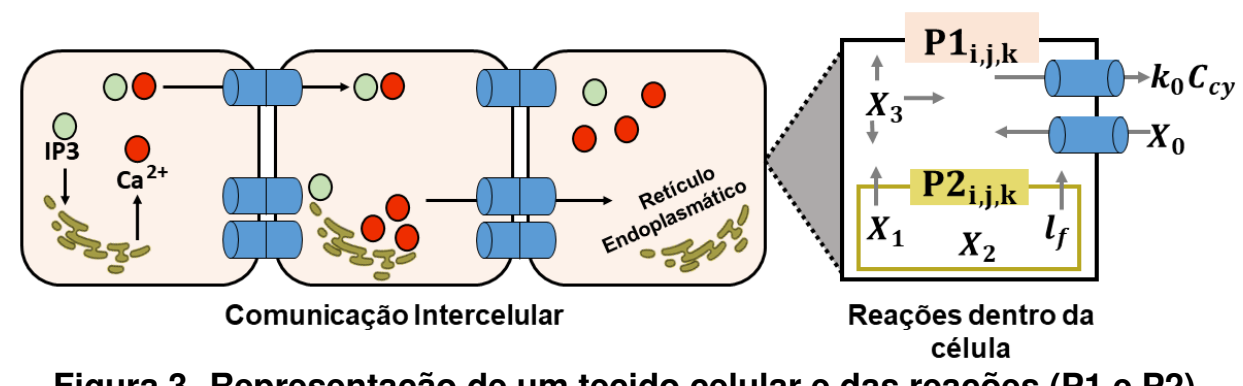

Figura 3. Representação de um tecido celular e das reações (P1 e P2)

Como o processo de difusão de $\mathrm{Ca}^{2+}$ ou $\mathrm{IP}_{3}$ ocorre através das junções comunicantes, as probabilidades $\left\{P\left(g_{1}\right), P\left(g_{2}\right), P\left(g_{3}\right)\right\}$ assumem as taxas de abertura e fechamento de cada conexônio, selecionadas pelo modelo estocástico com base nos estados $g_{1}, g_{2}$ e $g_{3}$ conforme especificado na Subseção 3.3.

\subsection{Modelo 3D do Tecido Astrócito}

Os astrócitos são células gliais, i.e., compõem o tecido nervoso junto com os neurônios e constituem aproximadamente metade das células do cérebro humano. Uma grande quantidade de evidências experimentais corrobora que os astrócitos participam da modulação e sincronização da atividade neuronal sináptica e das funções do sistema nervoso central que eram consideradas exclusivas dos neurônios. Estudos demonstram que os astrócitos propagam sinais intercelulares de $\mathrm{Ca}^{2+}$ por distâncias relativamente longas em resposta à estimulação por $\mathrm{IP}_{3}$, por isto este trabalho se propõe a investigar o uso dessas moléculas para comunicação molecular sintética no contexto dessas células. A propagação, neste caso, compreende duas etapas: a sinalização intracelular (dentro da célula) e a sinalização intercelular. A primeira resulta da estimulação interna via $\mathrm{IP}_{3}$ que leva à geração e/ou amplificação das concentrações de $\mathrm{Ca}^{2+}$ no citosol (líquido que preenche o interior do citoplasma da célula). A segunda envolve a propagação de moléculas de uma célula para suas células vizinhas. A difusão de íons $\mathrm{Ca}^{2+}$ e moléculas de $\mathrm{IP}_{3}$ é mediada por aberturas que conectam duas células pela área citosólica (junções comunicantes).

O modelo analítico da célula astrócito segue três dimensões (3D) com sinalização intracelular e intercelular. Para representar o tecido celular, considera-se uma área (A) composta de células. A distribuição das células na área segue uma organização em grade de três dimensões. Cada célula $\mathrm{c}_{i, j, k}$ é diferenciada neste modelo por seus índices $i, j$ e $k$, onde $i$ varia de 1 a $I ; j$ de 1 a $J$; e $k$ de 1 a $K$, sendo $I, J$ e $K$ a sua posição na grade 3D (matriz). As células podem se conectar a no máximo seis outras células vizinhas. As conexões entre as células seguem o estudo das topologias encontradas em astrócitos [Lallouette et al. 2014]. Considerado que cada célula contém um conjunto de reações internas (modeladas na Subseção 3.2) de pools P1 e P2, sendo P1 o fluxo do $\mathrm{IP}_{3}$ ou $\mathrm{Ca}^{2+}$ do citosol para espaço extracelular e P2 a difusão de $\mathrm{Ca}^{2+}$ do retículo endoplasmático para o citosol (Figura 3). O modelo estocástico de concentração (descrito posteriormente) calcula os valores de cada concentração de moléculas (pool) ao longo do tempo e executa as reações. O pool será negativamente ou positivamente afetado por uma constante $\alpha$ (Eq. 12) quando uma reação é executada.

\subsection{Modelo do Ruído Intracelular}

Esse artigo considera dois tipos de ruídos: intracelular (interno) e o intercelular (externo). Com relação ao ruído interno, ele é causado devido às constantes e aleatórias flutuações 
das concentrações de moléculas de $\mathrm{IP}_{3} \mathrm{e} \mathrm{Ca}^{2+}$. O ruído interno é modelado pelos termos $W_{1}$ e $W_{2}$ (Eq. 10 e Eq. 11) e toma como referência os eventos de reações químicas (Eqs. 1, 3 e 9) que ocorrem nas células astrócitos [Yu et al. 2009]. É amplamente aceito que a intensidade do ruído interno é proporcional à raiz quadrada do número de moléculas e inversamente proporcional ao volume da célula [He et al. 2017]. O ruído externo é decorrente da permeabilidade dos canais intercelulares (junções comunicantes), ele é gerado devido às flutuações na concentração de moléculas resultante das camadas adjacentes (células vizinhas), este ruído é detalhado na Subseção 4.1. Os termos $\mathrm{W}_{1} \mathrm{e} \mathrm{W}_{2}$ modelam o ruído conforme as Eqs. 1 e 9 . Na sequência, w $0_{i, j, k}$ e w $5_{i, j, k}$ são variáveis gaussianas independentes com médias nulas (zero) e variância unitária; $v$ é o volume da célula; $C_{i, j, k}-C_{m, n, l}$ representa a diferença de concentração de moléculas de $\mathrm{IP}_{3}$ ou $\mathrm{Ca}^{2+}$; e $\left\{P\left(g_{1}\right) P\left(g_{2}\right), P\left(g_{3}\right)\right\}$ representa a probabilidade de estados das junções comunicantes. $\mathrm{O}$ termo $\mathrm{W}_{2}$ modela o ruído incluindo a degradação do $\mathrm{IP}_{3}$, conforme Eq. 3.

$$
\begin{gathered}
W_{1}=\frac{1}{\sqrt{v}}\left(w 0_{i, j, k} \sqrt{X_{0}}-w 1_{i, j, k} \sqrt{k_{0} C_{c y}}+w 2_{i, j, k} \sqrt{X_{1}}-w 3_{i, j, k} \sqrt{X_{2}}\right. \\
\left.+w 4_{i, j, k} \sqrt{l_{f}}+\sum_{m, n, l, i, j, k} w 5_{i, j, k} \sqrt{C_{i, j, k}-C_{m, n, l}-\{} \mathrm{P}\left(\mathrm{g}_{1}\right) P\left(g_{2}\right), P\left(g_{3}\right)\right\} \\
W_{2}=\frac{1}{\sqrt{V}}\left(w_{3, i, j, k} \sqrt{X_{3}}-w_{4, i, j, k} \sqrt{I P_{K_{\text {deg }}}},\right.
\end{gathered}
$$

\subsection{Modelo Estocástico para Escolha das Reações}

Para determinar a concentração de moléculas de cada pool ao longo do tempo, as simulações seguem um modelo estocástico baseado no algoritmo de Gillespie [Gillespie 1977]. As simulações baseadas neste algoritmo produzem uma variabilidade precisa das reações químicas e servem para estudar os efeitos dos ruídos causados pelo comportamento estocástico inerente [Nakano and Liu 2010]. O modelo matemático estocástico executa o algoritmo de Gillespie para selecionar aleatoriamente uma célula a cada período de tempo. Em seguida, ele escolhe uma reação interna aleatória para a célula e programa um intervalo de tempo $(t)$ para essa reação. A execução de cada uma das reações $(R)$ requer um processo de agendamento dividido em duas fases: $(i)$ selecionar uma reação e $(i i)$ selecionar o valor de acréscimo de tempo. Para cada reação, é alocada uma constante de reação $\left(\mathrm{a}_{r}\right)$. Considerando que $\tau_{0}$ é a soma de todos as $\mathrm{a}_{r}$ de $R$, a próxima reação $\left(\mathrm{r}_{u}\right)$ escolhida é dada pela Eq. 12.

$$
r_{u}=M A X\left\{\frac{a_{r j}}{\tau_{0}}=\frac{a_{r j}}{\sum_{j=1}^{|R|} a_{r j}}\right\} .
$$

A escolha da reação é baseada no processo de seleção da roleta que seleciona os eventos probabilisticamente. Este processo é parcial com base nos valores de probabilidade das reações. Contudo, o processo de seleção $(u)$ deve satisfazer a Eq. 13.

$$
\sum_{j=1}^{u-1} \frac{\tau_{r j}}{\tau_{0}}<\rho_{1} \leq \sum_{j=1}^{u} \frac{\tau_{r j}}{\tau_{0}},
$$

em que $\rho_{1}$ é uma variável uniforme aleatória com valores binários $(0,1)$. Em cada intervalo de tempo $(t)$, um lapso de tempo $\left(\delta_{t}\right)$ é derivado com base no inicial $\tau_{0}$ conforme representado na Eq. 14.

$$
\tau_{0} . \delta_{t}=1 n \frac{1}{\rho_{2}}
$$


em que o termo $\rho_{2}$ representa uma variável binária uniforme. A condição final é $\sum_{t=0}^{|T|} \delta_{t}$ $<t_{0}$, onde $T$ é conjunto de $t$, e $t_{0}$ é o tempo de simulação predefinido. O resultado de executar uma reação é a mudança dos valores dos pools, i.e., de acordo com a equação diferencial, uma constante mudará o valor do conjunto conforme o resultado positivo ou negativo da reação executada. Em relação às reações intercelulares, quando estão agendadas para acontecer $a_{r}$ é substituído por $Z \Delta$, como observado na Eq. 9.

\section{Avaliação de Desempenho}

Esta seção descreve a metodologia empregada para analisar por simulação numérica o ruído intracelular e intercelular no modelo de comunicação molecular multiportadora. A definição dos parâmetros (Tabela 1) utilizados para as equações apresentadas na Seção 3 e as simulações seguem os valores de resultados de experimentações da literatura [Lavrentovich and Hemkin 2008, Venance et al. 1997, Goldbeter et al. 1990, Baigent et al. 1997, Höfer et al. 2002].

\begin{tabular}{|c|c|c|}
\hline Parâmetros & Símbolos & Valores \\
\hline Concentração de $\mathrm{Ca}^{2+}$ no citosol & $C_{c y}$ & $0.1 \mu M$ \\
\hline Concentração de $\mathrm{IP}_{3}$ & $C_{e r}$ & $1.5 \mu M$ \\
\hline Fluxo de $\mathrm{Ca}^{2+}$ do espaço extracelular para o citosol & $I P$ & $1.44 \mu M$ \\
\hline Vazamento do retículo para o citosol & $X_{0}$ & $0.05 \mu M$ \\
\hline Taxa de efluxo de $\mathrm{Ca}^{2+}$ do citosol para espaço extracelular & $k_{o}$ & $0.5 s^{-1}$ \\
\hline Vazamento do retículo para o citosol & $l_{f}$ & $0.5 s^{-1}$ \\
\hline Degradação do $\mathrm{IP}_{3}$ & $k_{\text {deg }}$ & $0.08 s^{-1}$ \\
\hline Fluxo máximo de $\mathrm{Ca}^{2+}$ no processo & $M_{2}$ & $15 \mu M / s$ \\
\hline Fluxo máximo de $\mathrm{Ca}^{2+}$ no processo e ${ }_{p}$ coeficiente de Hill & $M_{p}$ & $0.05 \mu \mathrm{M} / \mathrm{s}$ \\
\hline Fluxo para cálculo da produção de $\mathrm{IP}_{3}$ & $k_{p}$ & $0.3 \mu M$ \\
\hline Hill coeficiente & $n$ & 2.02 \\
\hline Variável de ativação de $\mathrm{IP}_{3}$ & $k_{I}$ & $0.15 \mu M$ \\
\hline Variável de inibição de $\mathrm{IP}_{3}$ & $k_{A}$ & $0.15 \mu M$ \\
\hline Valor máximo do fluxo de $\mathrm{Ca}^{2+}$ no citosol & $M_{3}$ & $40.0 s^{-1}$ \\
\hline Hill coeficiente & $m$ & 2.02 \\
\hline Coeficiente de difusão $\mathrm{Ca}^{2+}$ & $D_{C a^{2+}}$ & $350 \mu m^{2} / s$ \\
\hline Coeficiente de difusão $\mathrm{IP}_{3}$ & $D_{I P 3}$ & $280 \mu \mathrm{m} \mathrm{sec}-2$ \\
\hline Sensibilidade de uma junção à voltagem juncional & $\lambda$ & 0.37 \\
\hline Taxa de abertura do gap & $\vartheta_{j} m V$ & 90 \\
\hline Tensão juncional & $\vartheta_{0} m V$ & 60 \\
\hline Sensibilidade da junção à voltagem juncional (aberto) & $A \gamma\left(m V^{-1}\right)$ & 0.008 \\
\hline Sensibilidade da junção à voltagem juncional (fechado) & $A \beta\left(m V^{-1}\right)$ & 0.67 \\
\hline
\end{tabular}

Tabela 1. Parâmetros de Simulação

\subsection{Path Loss com Ruído Intercelular}

A perda de caminho ou Path loss é a redução da densidade de energia (atenuação) de uma onda portadora que se propaga através do espaço. Esta métrica inclui os valores do ganho de canal e permite a inclusão de outros efeitos para análise como por exemplo, a interferência e o desvanecimento de vários caminhos. Dessa forma, o Path loss (Eq. 15) foi aplicado para analisar o comportamento dos canais moleculares utilizando multiportadoras de informação (independente ou não independente) considerando o ruído do canal.

$$
\Gamma(f)=20 \log _{10}\left(\frac{\Gamma_{T}(f)}{\Gamma_{T_{0}}(f)}\right) .
$$


Os termos $\Gamma_{T}(f)$ e $\Gamma_{T_{0}}(f)$ representam respectivamente, a média e o valor inicial do pico de concentração, $(f)$ reflete a frequência em hertz $(\mathrm{Hz})$. A Equação 15 calcula a perda de caminho para uma molécula individual (i.e., para o inositol trifosfato $\Gamma^{I P_{3}}(f)$ ou para o cálcio $\Gamma^{C A^{2+}}(f)$ ). Portanto, ao usar multiportadoras a perda total de caminho é obtida por $\Gamma_{\text {total }}=\Gamma^{C a^{2+}}+\Gamma^{I P_{3}}+W_{3}$. Considerando que o canal depende das duas moléculas e o $W_{3}$ é o ruído do sistema, a soma dos $\operatorname{logs}\left(\Gamma_{\text {Total }}\right)$ segue:

$$
\Gamma_{\text {Total }}=\left(20 \log _{10}\left(\frac{\Gamma_{T}^{C a^{2+}}(f)}{\Gamma_{T_{0}}^{C a^{2+}}(f)}\right)\right)+\left(20 \log _{10}\left(\frac{\Gamma_{T}^{I P_{3}}(f)}{\Gamma_{T_{0}}^{I P_{3}}(f)}\right)\right)+W_{3} .
$$

em que os termos $\Gamma_{T}^{C a^{2+}}(f)$ e $\Gamma_{T}^{I P_{3}}(f)$ representam a concentração média de pico de $\mathrm{Ca}^{2+}$ e $\mathrm{IP}_{3}$, respectivamente; $\Gamma_{T_{0}}^{C a^{2+}}(f)$ e $\Gamma_{T_{0}}^{I P_{3}}(f)$ são o pico inicial de moléculas para $\mathrm{Ca}^{2+}$ e $\mathrm{IP}_{3}$, respectivamente. $W_{3}$ é o fator de ruído intercelular do sistema que satisfaz a Eq. 17.

$$
W_{3}= \begin{cases}0 & \text {, quando } I P_{3} \Perp C a^{2+}, \\ \left.N\left(\mu=0, X_{1}^{2}\right)\right|_{d B} & \text {, quando } I P_{3} \not \Perp C C a^{2+},\end{cases}
$$

onde $\Perp$ representa a independência e $\not \Perp$ não independência entre os dois tipos de moléculas $N\left(\mu, X_{1}^{2}\right)$ é uma distribuição normal, com média $\mu$ e variância $X_{1}^{2}$. Assim, é verificado se o receptor após uma distância de oito células mede a mesma concentração que a soma dos dois valores de saída correspondentes a uma única molécula, transmitida independentemente sem o ruído da outra molécula não enviada.

\subsection{Capacidade do Canal}

A capacidade do canal é o limite superior da taxa na qual a informação pode ser transmitida de forma confiável através de um canal de comunicação. Para a investigação da capacidade de informação, definiu-se probabilidades de transição de estados para o receptor e o transmissor. Para o transmissor é considerado: $(i)$ a estimulação e a liberação $\left(x=x_{1}\right)$ ou $(i i)$ silêncio $\left(x=x_{0}\right)$. Isso reproduz o processo de modulação usado em OOK, em que $x=x_{1}$ quando o bit transmitido representa o bit 1 e $x=x_{0}$ quando o bit é igual 0. Para o receptor são considerados outros dois estados: $(i)$ ativo i.e., quando o número de moléculas recebidas muda o estado da célula $\left(y=y_{1}\right)$, ou $(i i)$ inativo $\left(y=y_{0}\right)$, conforme a quantidade de moléculas recebidas. A transmissão de um bit único é discretizada em intervalos de tempo (duração do período de transmissão - Tb). Considerando os altos valores no período de transmissão, o que permite um tempo de sincronização muito mais alto comparado aos sistemas de comunicações convencionais. Assume-se que o receptor e o transmissor estão totalmente sincronizados e cientes de cada transmissão de bit, essa suposição é comum na literatura [Kilinc and Akan 2013, Nakano and Liu 2010, Barros et al. 2014, Pierobon and Akyildiz 2010]. A entropia de Shannon é usada em sistemas biológicos para representar o conteúdo de informação (em bits) em vários processos [Abshire and Andreou 2001].

$$
H(X)=-\sum_{x \in X}^{P}(x) \log _{2} P(x),
$$

onde $\mathrm{H}$ é a distribuição de probabilidades e $(X)$ é a quantidade informação associada a uma variável aleatória. Além disso, a entropia condicional $(X, Y)$ é definida com base na distribuição conjunta $p(x, y)$ e na distribuição condicional $p(x \mid y)$ de $x$ e $y$ :

$$
H(X \mid Y)=-\sum_{x \in X} \sum_{y \in Y} p(x, y) \log _{2} p(x \mid y),
$$




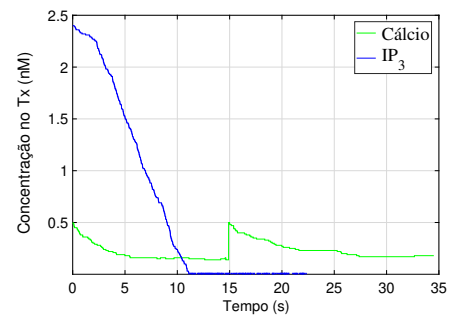

(a) $\mathrm{Tx}$

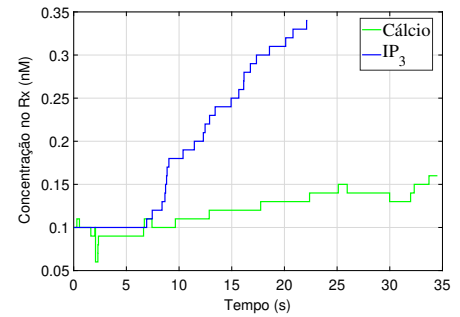

(b) $\mathrm{Rx}$

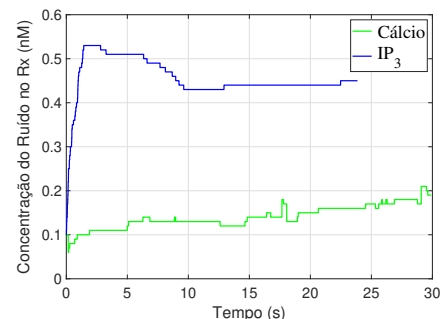

(c) Ruído Intracelular

Figura 4. Concentração espaço-temporal de moléculas

onde, $Y=\left\{y_{0}, y_{1}\right\}$. Todas as probabilidades restantes são definidas da seguinte forma: $p(x)=p\left(x=x_{0}\right)+p\left(x=x_{1}\right) ; p(y)=\left(p\left(y=y_{0}\right)+p\left(y=y_{1}\right)\right) * p(y \mid x) ; p\left(y=y_{0} \mid\right.$ $\left.x=x_{0}\right)=1-p\left(y=y_{1}|| x=x_{0}\right) ; p\left(y=y_{0} \mid x=x_{1}\right)=1-p\left(y=y_{1} \mid x=x_{1}\right)$.

Utilizou-se a informação mútua $I(X ; Y)$ para analisar a quantidade de informação que é transmitida do transmissor para o receptor. As probabilidades $p(x)$ e $p(y)$ representam a probabilidade de cada estado no transmissor ou no receptor, respectivamente. Como o período de transmissão é relativamente grande, os efeitos da memória na transmissão de bits são reduzidos, então assume-se que o canal é sem memória. $I(X ; Y)$ é baseado na entropia $H($.$) , e a capacidade segue a Eq. 20. Para calcular a independência entre as$ multiportadoras, a probabilidade de transmissão de um bit é a mesma para $\mathrm{IP}_{3} \mathrm{e} \mathrm{Ca}^{2+}$.

$$
C(X ; Y)=\max _{p(x)} I(X ; Y)=\sum_{y \in Y} \sum_{x \in X} p(x) p(y \mid x) \log _{2} \frac{p(y \mid x)}{p(y)} .
$$

\subsection{Resultados}

A Figura 4 apresenta o resultado de simulação numérica em Python para a oscilação da concentração de moléculas de $\mathrm{Ca}^{2+}$ e $\mathrm{IP}_{3}$ no Transmissor (Tx) e Receptor ( $\mathrm{Rx}$ ) e o ruído intracelular no RX versus tempo em um tecido celular de $3 \times(3 \times l) \times(20 \times l) \mu \mathrm{m}$ (micrômetros) de uma célula astrócito em que $l$ representa o comprimento de cada célula. Para as duas moléculas, a concentração inicial do Tx é $2 \times 10^{3} \mathrm{nM}$ e $500 \mathrm{nM}$ para o RX, a distância é de 8 células. A frequência de oscilação natural dos níveis de concentração em astrócitos é de $0.1 \mathrm{~Hz}$. A amplitude de oscilações é $2.5 \mu \mathrm{m}$ para o $\mathrm{IP}_{3}$ e $0.6 \mu \mathrm{m}$ para o $\mathrm{Ca}^{2+}$ medido pelo nível máximo de moléculas. Com base apenas nos níveis de concentração, observa-se comportamentos diferentes. Uma observação significativa está em como o $\mathrm{IP}_{3}$ afeta a sinalização intracelular de $\mathrm{Ca}^{2+}$. A estimulação de $\mathrm{IP}_{3}$ promove um aumento na concentração de $\mathrm{Ca}^{2+}$. As moléculas de $\mathrm{IP}_{3}$ estimulam a produção de $\mathrm{Ca}^{2+}$ por mais alguns segundos, mesmo quando a última molécula de $\mathrm{IP}_{3}$ já atingiu o $\mathrm{Rx}$. A Figura 4c apresenta o ruído intracelular no Rx. Conforme pode ser observado, o ruído intracelular da molécula de $\mathrm{Ca}^{2+}$ atinge uma concentração de 0.20 (nM) enquanto o ruído do $\mathrm{IP}_{3}$ atinge uma concentração máxima de $0.5(\mathrm{nM})$. Também foi possível identificar diferentes padrões entre os ruídos de cada molécula, enquanto o $\mathrm{IP}_{3}$ tende a estabilizar após 15 segundos e reduzir o valor de concentração, o cálcio apresenta mais varições e um ruído crescente. Isso é explicado pela constante estimulação de $\mathrm{Ca}^{2+}$ pela molécula $\mathrm{IP}_{3}$, como o processo de produção do $\mathrm{IP}_{3}$ é externo, o ruído aumenta muito rápido, mas a degradação também é maior.

A Figura 5 apresenta os resultados para a avaliação do Path loss (Figura 5a) e capacidade do canal (Figura 5b) com e sem ruído intercelular. Os resultados obtidos 
foram comparados com o desempenho da molécula de cálcio (única portadora), por ser a mais utilizada. Para o cálcio a concentração no Tx é de $2 \times 10^{3} \mathrm{nM}$ e de $1 \times 10^{5}$ nM para o Rx. Para o $\mathrm{IP}_{3}$, a concentração no Tx é de $2 \times 10^{3} \mathrm{nM}$ e a concentração inicial no transmissor é de $6 \times 10^{5} \mathrm{nM}$. Conforme pode ser observado na Figura $5 \mathrm{a}$ a pura propagação de $\mathrm{Ca}^{2+}$ apresenta uma perda de caminho maior do que o $\mathrm{IP}_{3}$ sozinho ou quando é empregado em conjunto com esta molécula. A medida que a distância entre o transmissor e o receptor aumenta, o Path loss também aumenta para todas as combinações afetando o desempenho. Comparando a perda de caminho em que cada molécula $\left(\mathrm{Ca}^{2+}\right.$ ou $\mathrm{IP}_{3}$ ) atua como portadora única de informação em distâncias de 1 a 8 células, a variação de perda durante o trajeto do $\mathrm{Ca}^{2+}$ é de aproximadamente $63.415 \%$, enquanto a variação do $\mathrm{IP}_{3}$ é de $33.274 \%$. Os resultados mostram uma perda de caminho mais suave para o $\mathrm{IP}_{3}$ quando a distância avaliada compreende entre 2 e 6 células, também é observado uma estabilidade ligeiramente superior ao $\mathrm{Ca}^{2+}$ ao longo de todo o caminho. O desempenho para o uso simultâneo das moléculas $\mathrm{IP}_{3} \Perp C a^{2+}$ e $\mathrm{IP}_{3} \not \Perp C a^{2+}$ está muito próximo dos resultados do uso isolado de $\mathrm{IP}_{3}$. Quando ocorre a paralelização dessas moléculas, obtém-se uma menor perda na transmissão, no entanto, uma perturbação indesejável na concentração do sinal molecular recebido pode ocorrer. Essa perturbação atua como um ruído, representado por $W_{3}$ quando $\mathrm{IP}_{3} \not \underline{L} \mathrm{Ca}^{2+}$. Contudo, os resultados mostraram que o ruído intercelular impacta pouco o path loss do canal.

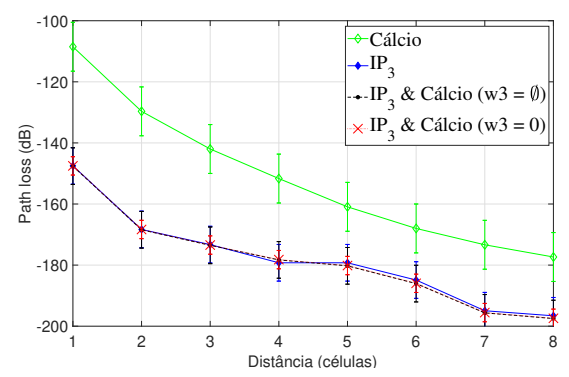

(a) Path loss

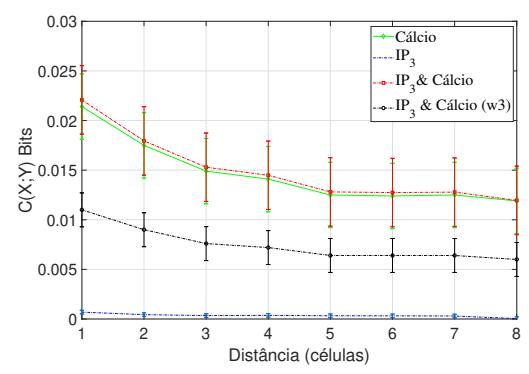

(b) Capacidade do canal

Figura 5. Path loss e Capacidade fim-a-fim em função da distância

A Figura 5b apresenta os resultados para a capacidade fim-a-fim em função da distância (em número de células). Os resultados da transmissão utilizando multiportadora com e sem ruído foram comparados com os resultados obtidos nas análises dos canais individuais de $\mathrm{Ca}^{2+}$ e $\mathrm{IP}_{3}$. A ativação do $\mathrm{Rx}$ depende de duas variáveis: a concentração de moléculas recebidas pelo estímulo externo e a concentração interna de moléculas. Quando as moléculas se distanciam do seu transmissor, sua concentração também diminui. A molécula de $\mathrm{Ca}^{2+}$ é a mais afetada pela distância e apresenta maior capacidade, já a molécula de $\mathrm{IP}_{3}$ apresenta valores baixos de capacidade, contudo é estável para distâncias maiores. A cooperação entre moléculas de forma independente reduz a perda de caminho e melhora a propagação de dados quando comparado com os resultados isolados de cada molécula nas duas métricas. Contudo ao considerar o ruído intercelular das moléculas $\mathrm{IP}_{3}$ $\not 1 \mathrm{Ca}^{2+}$, (i.e., quando uma molécula pode afetar o comportamento da outra) verificouse a degradação da capacidade de comunicação, esse resultado indica a necessidade do desenvolvimento de técnicas de filtragem de ruído. 


\section{Conclusão}

A comunicação molecular de curto alcance com base na sinalização de $\mathrm{IP}_{3} / \mathrm{Ca}^{2+}$ nos tecidos celulares é uma abordagem promissora para o desenvolvimento das nanorredes. Este artigo contribui com a caracterização e análise do ruído intracelular e intercelular em um modelo de comunicação molecular multiportadora. $\mathrm{O}$ modelo permitiu estudar o uso de moléculas $\mathrm{IP}_{3}$ e $\mathrm{Ca}^{2+}$ como multiportadoras e os ruídos em um modelo analítico 3D da célula astrócito. O ruído intercelular foi analisado sobre as métricas capacidade do canal e path loss. Os resultados mostraram que os ruídos afetam negativamente a capacidade do canal, embora tenha pouco impacto sobre a métrica path loss. Os resultado numéricos mostraram que os ruídos intracelulares tornam estocásticos os comportamentos dos canais. A análise permite uma melhor compreensão desse comportamento e pode auxiliar no desenvolvimento de filtros de ruídos. Os resultados também reforçam nossos resultados prévios que a cooperação entre esses dois canais, ou seja, $\mathrm{IP}_{3}$ e $\mathrm{Ca}^{2+}$, melhora a codificação e a transmissão de dados em comparação com o uso individual (única portadora). Em trabalhos futuros, pretende-se explorar e modelar novas fontes de ruídos da comunicação molecular, assim como desenvolver formas de maximizar o fluxo de informações utilizando filtros de ruídos.

\section{Agradecimentos}

Os autores agradecem o apoio da UFPR e auxílio financeiro do CNPq e da CAPES, processo 88882.382196/2019-01.

\section{Referências}

Abshire, P. and Andreou, A. G. (2001). Capacity and energy cost of information in biological and silicon photoreceptors. Proceedings of the IEEE (Journal), 89(7):1052-1064.

Akyildiz, I. F., Pierobon, M., Balasubramaniam, S., and Koucheryavy, Y. (2015). The internet of bio-nano things. IEEE Commun. Magazine, 53(3):32-40.

Baigent, S., Stark, J., and Warner, A. (1997). Modelling the effect of gap junction nonlinearities in systems of coupled cells. Journal of theoretical biology, 186(2):223-239.

Barros, M., Borges, L., Regis, C., Nogueira, M., and Loureiro, A. (2018a). Internet-dasbionano-coisas: Conectando-se às nanomáquinas. In Guidoni, D., editor, Livro dos Minicursos do SBRC 2018, pages 1-50. SBC.

Barros, M. T., Balasubramaniam, S., and Jennings, B. (2015). Comparative end-toend analysis of ca 2+-signaling-based molecular communication in biological tissues. IEEE Transactions on Communications, 63(12):5128-5142.

Barros, M. T., Balasubramaniam, S., Jennings, B., and Koucheryavy, Y. (2014). Transmission protocols for calcium-signaling-based molecular communications in deformable cellular tissue. IEEE Transactions on Nanotechnology, 13(4):779-788.

Barros, M. T., Borges, L. F., Régis, C. D. M., Nogueira, M., and Loureiro, A. (2018b). Internet-das-bionano-coisas: Conectando-se às nanomáquinas. In Guidoni, D. L., editor, Livro dos Minicursos do Simpósio Brasileiro de Redes de Computadores e Sistemas Distribuídos, chapter 2, pages 1-50. SBC.

Barros, M. T. and Dey, S. (2017). Set point regulation of astrocyte intracellular ca2+ signalling. In IEEE International Conference on Nanotechnology, pages 315-320.

Borges, L. F., Barros, M. T., and Nogueira, M. (2019). Explorando o potencial da molécula IP3 para a comunicação em nanorredes. In Anais do Simpósio Brasileiro de Redes de Computadores e Sistemas Distribuídos, pages 29-42. SBC. 
Borges, L. F., Barros, M. T., and Nogueira, M. (2020). A Multi-Carrier molecular communication model for astrocyte tissues. In 2020 IEEE International Conference on Communications (ICC): SAC Molecular, Biological, and Multi-Scale Communications Track, Dublin, Ireland.

Bukauskas, F. F., Bukauskiene, A., Bennett, M. V., and Verselis, V. K. (2001). Gating properties of gap junction channels assembled from connexin 43 and connexin 43 fused with green fluorescent protein. Biophysical journal, 81(1):137-152.

Decrock, E., De Bock, M., Wang, N., Gadicherla, A. K., Bol, M., Delvaeye, T., Vandenabeele, P., Vinken, M., Bultynck, G., Krysko, D. V., et al. (2013). Ip3, a small molecule with a powerful message. Biochimica et Biophysica Acta (BBA)-Molecular Cell Research, 1833(7):1772-1786.

Gillespie, D. T. (1977). Exact stochastic simulation of coupled chemical reactions. J. of physical chemistry, 81(25):2340-2361.

Goldbeter, A., Dupont, G., and Berridge, M. J. (1990). Minimal model for signal-induced ca2+ oscillations and for their frequency encoding through protein phosphorylation. Proceedings of the National Academy of Sciences, 87(4):1461-1465.

He, P., Nakano, T., Mao, Y., Lio, P., Liu, Q., and Yang, K. (2017). Stochastic channel switching of frequency-encoded signals in molecular communication networks. IEEE Communications Letters, 22(2):332-335.

He, P., Nakano, T., Mao, Y., Lio, P., Liu, Q., and Yang, K. (2018). Stochastic channel switching of frequency-encoded signals in molecular communication networks. IEEE Communications Letters, 22(2):332-335.

Heren, A. C., Kuran, M. S., Yilmaz, H. B., and Tugcu, T. (2013). Channel capacity of calcium signalling based on inter-cellular calcium waves in astrocytes. In The $3 \mathrm{rd}$ IEEE International Workshop on Molecular and Nano Scale Communication.

Höfer, T., Venance, L., and Giaume, C. (2002). Control and plasticity of intercellular calcium waves in astrocytes: a modeling approach. Journal of Neuroscience, 22(12):4850-4859.

Kilinc, D. and Akan, O. B. (2013). An information theoretical analysis of nanoscale molecular gap junction communication channel between cardiomyocytes. IEEE Transactions on Nanotechnology, 12(2):129-136.

Lallouette, J., De Pittà, M., Ben-Jacob, E., and Berry, H. (2014). Sparse short-distance connections enhance calcium wave propagation in a $3 \mathrm{~d}$ model of astrocyte networks. Frontiers in Comp. Neurosci., 8:45.

Lavrentovich, M. and Hemkin, S. (2008). A mathematical model of spontaneous calcium (ii) oscillations in astrocytes. J. of Theoretical Biology, 251(4):553-560.

Nakano, T. and Liu, J.-Q. (2010). Design and analysis of molecular relay channels: An information theoretic approach. IEEE Trans. on NanoBioscience, 9(3):213-221.

Nakano, T., Suda, T., Koujin, T., Haraguchi, T., and Hiraoka, Y. (2007). Molecular communication through gap junction channels: System design, experiments and modeling. In Bionetics, pages 139-146. IEEE.

Pierobon, M. and Akyildiz, I. F. (2010). A physical end-to-end model for molecular communication in nanonetworks. IEEE J. on Selected Areas in Comm., 28:602-611.

Venance, L., Stella, N., Glowinski, J., and Giaume, C. (1997). Mechanism involved in initiation and propagation of receptor-induced intercellular calcium signaling in cultured rat astrocytes. J. of Neuroscience, 17(6):1981-1992.

Yu, G., Yi, M., Jia, Y., and Tang, J. (2009). A constructive role of internal noise on coherence resonance induced by external noise in a calcium oscillation system. Chaos, Solitons \& Fractals, 41(1):273-283. 